\title{
END POINT ESTIMATES FOR RADON TRANSFORM OF RADIAL FUNCTIONS ON NON-EUCLIDEAN SPACES
}

\author{
ASHISHA KUMAR AND SWAGATO K. RAY
}

\begin{abstract}
We prove end point estimate for Radon transform of radial functions on affine Grasamannian and real hyperbolic space. We also discuss analogs of these results on the sphere.
\end{abstract}

\section{INTRODUCTION}

Given a function $f$ on $\mathbb{R}^{n}, n \geq 2$, the Radon transform of $f$ is defined by the formula

$$
R f(\omega, t)=\int_{\mathbb{R}^{n-1}} f\left(t \omega+y^{\prime}\right) d y^{\prime}, \quad \omega \in S^{n-1}, t \in \mathbb{R},
$$

whenever the above integral makes sense. One can analogously define the $d$-plane transform of a function as the integral of the function over $d$-dimensional planes with respect to the $d$-dimensional Lebesgue measure. Precisely, given $1 \leq d \leq n-1$ and a $d$-dimensional linear subspace $\Pi$ of $\mathbb{R}^{n}$, one defines

$$
T_{d} f(x, \Pi)=\int_{\Pi} f(x-y) d \lambda_{d}(y)
$$

where $\lambda_{d}$ denotes the $d$-dimensional Lebesgue measure on $\Pi$. Вy a simple application of Fubini's theorem one can see that the above integrals converge absolutely for $f \in L^{p}\left(\mathbb{R}^{n}\right)$, if $p=1$. But for $p>1$ the situation is rather involved. It was proved in $\left[23\right.$ that if $f \in L^{p}\left(\mathbb{R}^{n}\right), 1 \leq p<\frac{n}{d}$, then the lower dimensional integral (1.2) is well defined for allmost every $x$ and $\Pi$. Roughly speaking, the above phenomenon occurs because the $d$-plane transform is closely related to the Fourier transform and the Reisz potential. A natural question then is to ask: If $f \in L^{p}\left(\mathbb{R}^{n}\right), 1 \leq p<\frac{n}{d}$, then what can one say about the size of $T_{d} f$ ? Of course the size has to be measured with respect to the natural measure on the set of $d$-dimensional affine subspaces. The above problem was completely settled for $d=n-1$ in [17. It was shown in [17] that there exists a positive constant $C$ such that for all $f \in C_{c}^{\infty}\left(\mathbb{R}^{n}\right)$ the following inequality holds,

$$
\left(\int_{S^{n-1}}\left[\int_{\mathbb{R}}|R f(\omega, t)|^{q} d t\right]^{p^{\prime} / q} d \sigma_{n-1}(\omega)\right)^{1 / p^{\prime}} \leq C\|f\|_{L^{p}\left(\mathbb{R}^{n}\right)},
$$

$1 \leq p<n /(n-1), 1 / q=(n / p)-n+1,1 / p^{\prime}=1-(1 / p)$. Here $\sigma_{n-1}$ denotes the normalized rotation invariant measure on the sphere.

Apart from the above result another intriguing observation related to the end-point estimate of (1.3) involving Lorentz norm was made in [17. It was shown in [17 that the end-point estimate of (1.3) holds for $n \geq 3$, that is,

$$
\left(\int_{S^{n-1}}\left(\sup _{t \in \mathbb{R}}|R f(\omega, t)|\right)^{n} d \sigma_{n-1}(\omega)\right)^{1 / n} \leq C_{n}\|f\|_{L^{\frac{n}{n-1}, 1}\left(\mathbb{R}^{n}\right)}
$$

2000 Mathematics Subject Classification. Primary 44A12, 42B20; Secondary 31B99.

Key words and phrases. Radon transform, $d$-plane transform, Lorentz Spaces, Hyperbolic space, Sphere, Affine Grassmann. 
But the above estimate fails for the case $n=2$. A consequence of the above is that if $f \in L^{\frac{n}{n-1}}, 1\left(\mathbb{R}^{n}\right), n \geq$ 3 then its Radon transform is well defined for almost every hyperplane. The failure of the end-point estimate for $n=2$ can be attributed to the existence of compact Kakeya sets in $\mathbb{R}^{2}$ of arbitrary small Lebesgue measure ([17], p. 642). Since the indicator function of a set with radial symmetry and arbitrarily small $L^{2,1}$ norm cannot contain line segments of a fixed length in every direction, one can still hope for an end-point estimate for the radial functions in the case $n=2$. This viewpoint was adopted in [5] and the question regarding the end-point estimate for the $d$-plane transform of radial functions was answered: if $1 \leq d \leq n-1$ then for all radial functions $f$ the following estimate holds,

$$
\sup _{(x, \Pi) \in \mathbb{R}^{n} \times G_{n, d}}\left|T_{d} f(x, \Pi)\right| \leq C\|f\|_{L^{\frac{n}{d}, 1}\left(\mathbb{R}^{n}\right)} .
$$

Here $G_{n, d}$ stands for the set of $d$-dimensional linear subspaces of $\mathbb{R}^{n}$ and $C>0$ is a constant independent of $f$. In this paper our main objective is to establish analogs of (1.5) on affine Grassmannian, real hyperbolic space and the sphere.

There is a wealth of information available in the literature on the study of $d$-plane transform on affine Grassmannian (see for instance [7, 8, 9, 10, 24]). However, the study of $d$-plane transform of $L^{p}$ functions on affine Grassmannian seems to be relatively new. Investigation regarding $L^{p}-L^{q}$ mapping property of the $d$-plane transform for affine Grassmannian was recently initiated in 22. This notion of $d$-plane transform generalizes the notion of $d$-plane transform of functions defined earlier. This motivated us to look for an estimate similar to (1.5) for the $d$-plane transform of radial functions on affine Grassmannian. Our main result in section 2 shows that it is possible to prove an appropriate analogue of (1.5) for generalized $d$-plane transform of radial functions on affine Grassmannian (see Theorem 2.2).

Next we turn towards the real hyperbolic space. There are several papers which deal with $L^{p}-L^{q}$ mapping property of the totally geodesic radon transform on real hyperbolic spaces (see for instance [1, 2, 14, 25] and references there in). But none of these papers address the question of end point estimates for the Radon transform. The best possible $L^{p}-L^{q}$ mapping property of the Radon transform (or more generally the $d$-plane transform) on these spaces seem to be a hard problem. However, it turns out that the same problem on the restricted class of radial functions is not very difficult to tackle. In fact, analogues of (1.5) has already appeared in the non Euclidean setup. The first one is by Cowling, Meda and Setti [4. While working on Kunze-Stein phenomena on homogeneous trees they proved that the horospherical Radon transform of radial functions defines a continuous operator from $L^{2,1}$ to $L^{\infty}$ ([4], Theorem 2.5). The second result is by Ionescu [13]. It was shown in [13] that for rank one Riemannian symmetric spaces of non compact type the horospherical Radon transform of radial functions is also continuous from $L^{2,1}$ to $L^{\infty}$ ([13, Proposition 2).

These results motivated us to consider the $d$-dimensional totally geodesic Radon transform of radial functions on real hyperbolic space $\mathbb{H}^{n}$ and the sphere $S^{n}$. A result in 1] says that: If $f \in L^{p}\left(\mathbb{H}^{n}\right)$, $1 \leq p<(n-1) /(d-1)$, then $f$ is integrable over almost every $d$-dimensional totally geodesic submanifold. In analogy with (1.5) it is natural to enquire about the validity of the end-point estimate only for radial functions. We answer the question in Section 3 as follows (see Theorem 3.9): the $d$-plane transform restricted to the class of radial functions defines a continuous linear map from $L^{\frac{n-1}{d-1}, 1}\left(\mathbb{H}^{n}\right)$ to $L^{\infty}\left(\mathbb{R}^{+}\right)$ if $n \geq 3$. As a consequence it follows that if $f$ is radial and $f \in L^{\frac{n-1}{d-1}, 1}\left(\mathbb{H}^{n}\right), n \geq 3$, then $f$ is integrable over almost every $d$-dimensional totally geodesic submanifold. This is in the same spirit as $\mathbb{R}^{n}$, homogeneous trees and rank one symmetric spaces of non compact type. However there are some non Euclidean consequences of the above result (see Corollary 3.11). One such is that if $f \in L^{\frac{n-1}{d-1}, 1}\left(\mathbb{H}^{n}\right)$ 
and is radial then its Radon transform has an exponential decay at infinity. As a consequence we will see that the $d$-dimensional totally geodesic Radon transform of radial functions is also continuous from $L^{\frac{n-1}{d-1}, 1}\left(\mathbb{H}^{n}\right)$ to $L^{n-1, \infty}\left(\mathbb{R}^{+}\right)$. This is in sharp contrast with the Euclidean spaces.

In Section 4 we consider the case of the sphere. The situation here is very different because of compactness. It is known from [20] that in this case the $d$-plane transform is continuous from $L^{p}\left(S^{n}\right)$ to $L^{p}(S O(n+1) / S O(n-d) \times S O(d+1)), 1 \leq p \leq \infty$ (see also [25]). Here the quotient space $S O(n+1) / S O(n-d) \times S O(d+1)$ is viewed as the space of $d$-dimensional totally geodesic submanifolds of $S^{n}$. In this case one can show that the exact analogue of (1.5), for functions which are invariant under the action of $S O(n)$, is not true (Example 4.6). It turns out that one can prove a result analogous to (1.5) if the $S O(n+1)$ invariant measure on $S O(n+1) / S O(n-d) \times S O(d+1)$ is considered along with a weight which is naturally associated with the structure of the set of $d$-dimensional totally geodesic submanifolds (see Theorem 4.7).

\section{Affine Grassmannian}

2.1. Notation and Preliminaries. Let $G_{n, k}$ be the standard Grassmann manifold of all $k$-dimensional linear subspaces of $\mathbb{R}^{n}, 0 \leq k<n$. The rotation group $S O(n)$ acts on $G_{n, k}$ by $A \cdot \xi=A(\xi), A \in$ $S O(n), \xi \in G_{n, k}$. The above action can easily be seen to be transitive and moreover the isotropy subgroup at $\xi_{0}=\operatorname{span}\left\{e_{1}, \ldots, e_{k}\right\}$ is $S O(k) \times S O(n-k)$ (see [16], p. 140). Consequently we can identify $G_{n, k}$ with the compact homogeneous space $S O(n) / S O(k) \times S O(n-k)$. This identification allows us to talk about the $S O(n)$ invariant measure on $G_{n, k}$ with total mass 1 . This measure will be denoted by $d \xi$. Let $G(n, k)$ denotes the set of all $k$-dimensional affine subspaces of $\mathbb{R}^{n}$. Given $\xi \in G_{n, k}$ and $u \in \xi^{\perp}$ the translated plane $\xi+u \in G(n, k)$. Moreover, given any $\tau \in G(n, k)$ there exists unique $\xi \in G_{n, k}$ and unique $u \in \xi^{\perp}$ such that $\tau=\xi+u$. Consequently, $G(n, k)$ can be parameterized by the pair $(\xi, u), \xi \in G_{n, k}, u \in \mathbb{R}^{n-k}$. The manifold $G(n, k)$ can also be viewed as a homogeneous space of the Euclidean motion group $M(n)=O(n) \times \mathbb{R}^{n}$. Given an element $(T, v) \in M(n)$ and $\left.\tau \in G_{(} n, k\right)$ we define

$$
(T, v) \cdot \tau=\left\{\left(\begin{array}{cc}
T & v \\
0 & 1
\end{array}\right)\left(\begin{array}{c}
w \\
1
\end{array}\right) \mid w \in \tau\right\}
$$

This action is known to be transitive. Let $\left\{e_{1}, e_{2}, \ldots, e_{n}\right\}$ be the standard orthonormal basis of $\mathbb{R}^{n}$ and $\tau_{k}=\operatorname{Re}_{1} \oplus \ldots \oplus \operatorname{Re}_{k}$ (we assume $\tau_{k}=0$ if $k=0$. It then follows that the isotropy subgroup $H_{k}$ at $\tau_{k}$ is given by

$$
\begin{aligned}
H_{k} & =\left\{(T, v) \in M(n):(T, v) \cdot \tau_{k}=\tau_{k}\right\} \\
& =\left\{\left(\begin{array}{ccc}
T 1 & 0 & v \\
0 & T_{2} & 0 \\
0 & 0 & 1
\end{array}\right): T_{1} \in O(k), T_{2} \in O(n-k), v \in \tau_{k}\right\} .
\end{aligned}
$$

Hence $G(n, k) \approx M(n) /(O(k) \times O(n-k)) \times \mathbb{R}^{k}$.

Let $\xi$ be a $d$-plane, $k<d<n$, and $\tau$ a $k$-plane with $\tau \subset \zeta$. According to our convention we have $\zeta=(\eta, v), \tau=(\xi, u)$ where $\eta \in G_{n, d}, \xi \in G_{n, k}, v \in \eta^{\perp}$ and $u \in \zeta^{\perp}$. Since $\tau \subset \xi$ it then follows immediately that $\xi \subset \eta$. We claim that there exists $x \in \xi^{\perp} \cap \eta$ such that $u=v+x$. Let $A \in \tau$ then $A=Z+u=W+v, Z \in \xi, W \in \eta, u \in \xi^{\perp}, v \in \eta^{\perp}$. Hence $u-v=W-Z \in \eta$. On the other hand $\eta^{\perp} \subset \xi^{\perp}$ (as $\xi \subset \eta$ ) and hence $u-v \in \xi^{\perp}$. If we choose $x=u-v$ then we are through. We consider the product measure $d \mu_{k}(\tau)=d \xi d u$ on $G(n, k)$, where $d \xi$ is the measure on $G_{n, k}$ and $d u$ is the $(n-k)$-dimensional Lebesgue measure on $\mathbb{R}^{n-k}$. For $n>d>k$ and $\eta \in G_{n, d}$, we denote $G_{k}(\eta)$ the 
Grassmann manifold of $k$-dimensional linear subspaces of $\eta$. We write

$$
\tau=(\xi, u) \in G(n, k), \quad u \in \xi^{\perp} ; \quad \zeta=(\eta, v) \in G(n, d), \quad v \in \eta^{\perp} .
$$

The $d$-plan transform of a function $f$ on $G(n, k)$ is a function $R_{d} f$ on $G(n, d)$ defined by

$$
R_{d} f(\zeta)=\int_{\tau \subset \zeta} f(\tau)=\int_{\xi \subset \eta} d_{\eta} \xi \int_{\xi^{\perp} \cap \eta} f(\xi, v+x) d x .
$$

Here $d_{\eta} \xi$ denotes the normalized measure on Grassmannian $G_{k}(\eta)$ of all $k$-dimensional linear subspaces of $\eta$ and $d x$ denotes the $(d-k)$-dimensional Lebesgue measure.

If $\tau=(\xi, u) \in G(n, k)$, the distance $\|u\|$ of the plane $\tau$ from the origin will be denoted by $|\tau|$. A function $f$ defined on $G(n, k)$ is called radial if $f(\xi, u)=f\left(\xi^{\prime}, u^{\prime}\right)$ whenever $\|u\|=\left\|u^{\prime}\right\|$. This means that a radial functions depends only on the distance of the $k$-plane from the origin. For radial functions the formula (2.7) can be written in a more concrete form. Since $v \in \eta^{\perp}$ and $x \in \eta$ it follows from (2.7) that if $f$ is radial then

$$
R_{d} f(\zeta)=R_{d} f(\eta, v)=C_{n, d, k} \int_{\mathbb{R}^{(d-k)}} f\left(\left(\|v\|^{2}+\|x\|^{2}\right)^{1 / 2}\right) d x .
$$

This implies that $R_{d} f$ is also a radial function on $G(n, d)$. By writing $\|v\|=s$ and using polar coordinates on $\mathbb{R}^{d-k}$ we get the expression

$$
R_{d} f(\eta, v)=A_{d} f(s)=C_{n, d, k} \int_{s}^{\infty} f(t)\left(t^{2}-s^{2}\right)^{\frac{d-k}{2}-1} t d t .
$$

This formula will play a crucial role in the main result of this section.

We will need the notion of Lorentz spaces in the present as well as in the subsequent sections. We briefly recall some relevant results on Lorentz spaces (see [6, 26] for details). Let $(M, m)$ be a $\sigma$-finite measure space, $f: M \longrightarrow \mathbb{C}$ be a measurable function and $p \in[1, \infty), q \in[1, \infty]$. We define

$$
\|f\|_{p, q}^{*}= \begin{cases}\left(\frac{q}{p} \int_{0}^{\infty}\left[f^{*}(t) t^{1 / p}\right]^{q} \frac{d t}{t}\right)^{1 / q} & \text { when } q<\infty \\ \sup _{t>0} t d_{f}(t)^{1 / p} & \text { when } q=\infty .\end{cases}
$$

Here $d_{f}$ is the distribution function of $f$ and $f^{*}(t)=\inf \left\{s \mid d_{f}(s) \leq t\right\}$ is the nonincreasing rearrangement of $f\left(\left[6\right.\right.$, p. 45]). We take $L^{p, q}(M)$ to be the set of all measurable $f: M \longrightarrow \mathbb{C}$ such that $\|f\|_{p, q}^{*}<\infty$. By $L^{\infty, \infty}(M)$ and $\|\cdot\|_{\infty, \infty}$ we mean respectively the space $L^{\infty}(M)$ and the norm $\|\cdot\|_{\infty}$. For $p, q \in[1, \infty)$ the following identity gives an alternative expression of $\|\cdot\|_{p, q}^{*}$ which we will use.

$$
\frac{q}{p} \int_{0}^{\infty}\left(t^{1 / p} f^{*}(t)\right)^{q} \frac{d t}{t}=q \int_{0}^{\infty}\left(t d_{f}(t)^{1 / p}\right)^{q} \frac{d t}{t}
$$

The proof of this identity can be found, for instance, in [19]. For $p, q$ in the range above, $L^{p, p}(M)=$ $L^{p}(M)$ and if $q_{1} \leq q_{2}$ then $\|f\|_{p, q_{2}}^{*} \leq\|f\|_{p, q_{1}}^{*}$ and consequently $L^{p, q_{1}}(M) \subset L^{p, q_{2}}(M)$. It is easy to see from above that for the indicator function of a measurable set of finite measure $E$ we have $\left\|\chi_{E}\right\|_{L^{p, 1}(M)}=m(E)^{1 / p}$.

In this section and everywhere else we will follow the standard practice of using the letter $C$ for constant, whose value may change from one line to another. Occasionally the constant $C$ will be suffixed to show its dependency on important parameters. We will also use the symbol $f(x) \asymp g(x)$ to mean that there exist two positive constants $C_{1}, C_{2}$ such that $C_{1} f(x) \leq g(x) \leq c_{2} f(x)$ for appropriate values of $x$. 
2.2. Main result. Regarding the estimate of $R_{d} f$ for $L^{p}$ functions on $G(n, k)$ it is known from [22], Corallary 2.6 that if $f \in L^{p}(G(n, k)), 1 \leq p<\frac{n-k}{d-k}$ then the integrals involved in (2.7) is well defined. This range of $p$ is sharp in the sense that if $p \geq \frac{n-k}{d-k}$ then there exists a radial function $f \in L^{p}(G(n, k))$ such that $R_{d} f(\zeta)=\infty$ for almost every $\zeta \in G(n, d)$. Our main result in this section deals with behaviour of $R_{d}$ restricted to radial functions in $L^{\frac{n-k}{d-k}, 1}(G(n, k))$. We start by quoting a simple lemma from [15].

Lemma 2.1. Let $n \in \mathbb{N}$ and $\gamma \geq 1$. If $x_{1} \geq x_{2} \geq \ldots \geq x_{n} \geq 0$ are real numbers then the following inequality holds,

$$
\left(\sum_{i=1}^{n}(-1)^{i-1} x_{i}\right)^{\gamma} \leq \sum_{i=1}^{n}(-1)^{i-1}\left(x_{i}\right)^{\gamma}
$$

The main result of this section is the following Theorem.

Theorem 2.2. If $n>d>k \geq 0$, then there exists a constant $C>0$ such that for all radial functions $f$ on $G(n, k)$ the following estimate holds

$$
\sup _{s \in(0, \infty)} A_{d}(f)(s) \leq C\|f\|_{L^{\frac{n-k}{d-k}, 1}(G(n, k))} .
$$

Proof. In view of general theory of Lorentz spaces it suffices to prove (2.11) for indicator functions of radial open sets with finite measure (see [26], Theorem 3.13 and [5]). Since these functions can be approximated by functions of form $\chi_{\cup_{i=1}^{l} E_{i}}$ where $E_{i}=\left\{\tau \in G(n, k): a_{i} \leq|\tau|<b_{i}\right\}, b_{i}<a_{i+1}, a_{1} \geq 0$ and $i=1, \ldots, l$ it suffices to prove the result for these functions. An explicit calculation using (2.9) shows that

$$
A_{d}\left(\chi_{E_{i}}\right)(s)=C \begin{cases}{\left[\left(b_{i}^{2}-s^{2}\right)^{\frac{d-k}{2}}-\left(a_{i}^{2}-s^{2}\right)^{\frac{d-k}{2}}\right],} & s<a_{i} \\ \left(b_{i}^{2}-s^{2}\right)^{\frac{d-k}{2}}, & a_{i} \leq s \leq b_{i} \\ 0, & b_{i}<s\end{cases}
$$

where $s=|\zeta|, \zeta \in G(n, d)$. If we denote $E=\cup_{i=1}^{l} E_{i}$ then by using linearity of $A_{d}$ and (2.12) we get that

$$
A_{d}\left(\chi_{E}\right)(s)=C\left\{\begin{array}{l}
\sum_{i=1}^{l}\left[\left(b_{i}^{2}-s^{2}\right)^{\frac{d-k}{2}}-\left(a_{i}^{2}-s^{2}\right)^{\frac{d-k}{2}}\right], \quad \text { if } s<a_{1} . \\
\left(\left(\sum_{i=j+1}^{l}\left[\left(b_{i}^{2}-s^{2}\right)^{\frac{d-k}{2}}-\left(a_{i}^{2}-s^{2}\right)^{\frac{d-k}{2}}\right]\right)+\left(b_{j}^{2}-s^{2}\right)^{\frac{d-k}{2}}\right) \\
\quad \text { if } a_{j} \leq s<b_{j}, 1 \leq j \leq l . \\
\sum_{i=j+1}^{l}\left[\left(b_{i}^{2}-s^{2}\right)^{\frac{d-k}{2}}-\left(a_{i}^{2}-s^{2}\right)^{\frac{d-k}{2}}\right] \\
\quad \text { if } b_{j} \leq s<a_{j+1}, 1 \leq j \leq l-1 . \\
0, \quad b_{l}<s .
\end{array}\right.
$$

Now, for a fixed $s>0$, let $E_{s}$ denotes the set $\{\tau \in E:|\tau|>s\}$ so that $\chi_{E_{s}} \leq \chi_{E}$ and hence the corresponding distribution functions satisfy the relation $d_{\chi_{E_{s}}}(r) \leq d_{\chi_{E}}(r)$ for all $r>0$. This implies that $\left\|\chi_{E_{s}}\right\|_{L^{\frac{n-k}{d-k}, 1}(G(n, k))} \leq\left\|\chi_{E}\right\|_{L^{\frac{n-k}{d-k}, 1}(G(n, k))}$. To prove the theorem it thus suffices to show that for all $s>0$

$$
A_{d}\left(\chi_{E}\right)(s) \leq C\left\|\chi_{E_{s}}\right\|_{L^{\frac{n-k}{d-k}, 1}(G(n, k))},
$$

where $C$ is independent of $s$ and $l$. 
We now turn towards the calculation of the required Lorentz norm of the function $\chi_{E_{s}}$. By using polar coordinates on $\mathbb{R}^{n-k}$ and the expression of the measure on $G(n, k)$ given in the beginning of this section it follows that

$$
\begin{aligned}
& \left\|\chi_{E_{s}}\right\|_{L^{\frac{n-k}{d-k}, 1}(G(n, k))}=\mu_{k}\left(E_{s}\right)^{\frac{d-k}{n-k}} \\
& =C\left\{\begin{array}{l}
\left(\sum_{i=1}^{l}\left[b_{i}{ }^{n-k}-a_{i}{ }^{n-k}\right]\right)^{\frac{d-k}{n-k}}, \quad \text { if } s<a_{1} . \\
\left(\left(\sum_{i=j+1}^{l}\left[b_{i}{ }^{n-k}-a_{i}{ }^{n-k}\right]\right)+b_{j}{ }^{n-k}-s^{n-k}\right)^{\frac{d-k}{n-k}}, \\
\quad \text { if } a_{j}<s<b_{j}, 1 \leq j \leq l . \\
\left(\sum_{i=j+1}^{l}\left[b_{i}{ }^{n-k}-a_{i}{ }^{n-k}\right]\right)^{\frac{d-k}{n-k}}, \\
\quad \text { if } b_{j}<s<a_{j+1}, 1 \leq j \leq l-1 . \\
0, \quad \text { if } b_{l}<s .
\end{array}\right.
\end{aligned}
$$

We will now consider $s \in\left(a_{j}, b_{j}\right)$ for a fixed $j$ and prove (2.14). Other cases can be dealt with exactly the same way.

For $a>0, \gamma \geq 1$ and $x \geq y \geq 0$ we apply Lemma 2.1 to get the inequality $(x+a)^{\gamma}-x^{\gamma} \geq(y+a)^{\gamma}-y^{\gamma}$. If we now choose $\gamma=(n-k) / 2$ (which is greater than or equal to 1 by hypothesis) and $x=a_{i}^{2}$, $y=a_{i}^{2}-s^{2}, a=b_{i}^{2}-a_{i}^{2}$ and use the previous inequality then we get

$$
b_{i}^{n-k}-a_{i}{ }^{n-k} \geq\left[\left(b_{i}^{2}-s^{2}\right)^{\frac{n-k}{2}}-\left(a_{i}^{2}-s^{2}\right)^{\frac{n-k}{2}}\right] .
$$

Similarly,

$$
b_{j}{ }^{n-k}-s^{n-k} \geq\left(b_{j}^{2}-s^{2}\right)^{\frac{n-k}{2}} .
$$

Hence,

$$
\begin{aligned}
& \left(\sum_{i=j+1}^{l}\left[b_{i}{ }^{n-k}-a_{i}{ }^{n-k}\right]+b_{j}{ }^{n-k}-s^{n-k}\right)^{\frac{d-k}{n-k}} \\
& \geq\left(\sum_{i=j+1}^{l}\left[\left(b_{i}^{2}-s^{2}\right)^{\frac{n-k}{2}}-\left(a_{i}^{2}-s^{2}\right)^{\frac{n-k}{2}}\right]+\left(b_{j}^{2}-s^{2}\right)^{\frac{n-k}{2}}\right)^{\frac{d-k}{n-k}},
\end{aligned}
$$

for all $s \in\left(a_{j}, b_{j}\right)$. As $\left(b_{l}^{2}-s^{2}\right)^{(n-k) / 2}>\left(a_{l}^{2}-s^{2}\right)^{(n-k) / 2}>\ldots>\left(b_{j}^{2}-s^{2}\right)^{(n-k) / 2}$ we can apply Lemma 2.1 for $\gamma=(n-k) /(d-k)$ in (2.16) to get

$$
\begin{aligned}
& \left(\sum_{i=j+1}^{l}\left[\left(b_{i}^{2}-s^{2}\right)^{\frac{n-k}{2}}-\left(a_{i}^{2}-s^{2}\right)^{\frac{n-k}{2}}\right]+\left(b_{j}^{2}-s^{2}\right)^{\frac{n-k}{2}}\right)^{\frac{d-k}{n-k}}, \\
& \geq\left(\left(\sum_{i=j+1}^{l}\left[\left(b_{i}^{2}-s^{2}\right)^{\frac{d-k}{2}}-\left(a_{i}^{2}-s^{2}\right)^{\frac{d-k}{2}}\right]\right)+\left(b_{j}^{2}-s^{2}\right)^{\frac{d-k}{2}}\right) .
\end{aligned}
$$

The result now follows from (2.16) and the last inequality.

Remark 2.3. We note that if $k=0$ then the estimate (2.11) boils down to the estimate (1.5) proved in [5].

We will now briefly discuss the $L^{p}-L^{q}$ mapping property of $A_{d}$. We first recall that $R_{d}$ satisfies the trivial estimate $\left\|R_{d} f\right\|_{L^{1}(G(n, d))} \leq C\|f\|_{L^{1}(G(n, k))}$ (see [22]). We can thus use (2.11) and off-diagonal Marcinkiewicz interpolation theorem ([6], Theorem 1.4.19) to obtain the following result. 
Theorem 2.4. If $0 \leq k<d \leq n-1,1 \leq p<\frac{n-k}{d-k}$ and $\frac{n-k}{p}=d-k+\frac{n-d}{q}$, then there exists a constant $C>0$ such that for all radial functions $f \in L^{p}(G(n, k))$ the following inequality holds,

$$
\left\|A_{d} f\right\|_{L^{q}(G(n, d))} \leq C\|f\|_{L^{p}(G(n, k))} .
$$

\section{Real Hyperbolic Space}

3.1. Notation and Preliminaries. In this section we will set up notation and explain the basic facts needed to deal with the Radon transform on real hyperbolic space. Most of these material is standard and can be found in [1, 2], [3, [11, [18, 21], 27].

For $n \geq 2, \mathbb{E}^{n, 1}$ denotes the set $\mathbb{R}^{n+1}$ equipped with the bilinear form

$$
[x, y]=-x_{1} y_{1}-\cdots-x_{n} y_{n}+x_{n+1} y_{n+1}, \quad x, y \in \mathbb{E}^{n, 1} .
$$

We consider the set $F^{n}=\left\{x \in \mathbb{E}^{n, 1}:[x, x]=1\right\}$. The set $F^{n}$ is a hyperboloid of two sheets. The $n$-dimensional real hyperbolic space $\mathbb{H}^{n}$ is defined as the upper sheet of the hyperboloid, that is,

$$
\mathbb{H}^{n}=\left\{x \in \mathbb{E}^{n, 1}:[x, x]=1, x_{n+1}>0\right\} .
$$

Let $O(n, 1)$ denotes the group of invertible linear transformations of $\mathbb{R}^{n+1}$ which preserves the bilinear form given in (3.18), that is,

$$
\begin{aligned}
O(n, 1) & =\left\{g \in G L(n+1, \mathbb{R}):[g x, g y]=[x, y] \text { for all } x, y \in \mathbb{E}^{n, 1}\right\} \\
& =\left\{g \in G L(n+1, \mathbb{R}): g^{t} J g=g J g^{t}=J\right\},
\end{aligned}
$$

where $J=\operatorname{diag}(-1, \ldots,-1,1)$ ([18, Theorem 3.1.4). We define $S O(n, 1)=\{g \in O(n, 1): \operatorname{det} g=1\}$. Let $O_{0}(n, 1)$ denotes the subgroup of $O(n, 1)$ given by,

$$
O_{0}(n, 1)=\left\{g \in O(n, 1): e_{n+1}^{t} g e_{n+1}>0\right\} .
$$

The subgroup $S O_{0}(n, 1)$ of $O(n, 1)$ is defined by,

$$
S O_{0}(n, 1)=O_{0}(n, 1) \cap S O(n, 1) .
$$

It is known that the natural action of $S O_{0}(n, 1)$ on $\mathbb{H}^{n}$ is transitive (18, Theorem 3.1.6). Let $\left\{e_{1}, \ldots, e_{n+1}\right\}$ be the standard orthonormal basis of $\mathbb{R}^{n+1}$ and $x_{0}=e_{n+1}$ be the origin of $\mathbb{H}^{n}$. Let $K$ be the isotropy subgroup of $S O_{0}(n, 1)$ at $x_{0}$, that is, $K=\left\{g \in S O_{0}(n, 1): g\left(x_{0}\right)=g\left(e_{n+1}\right)=x_{0}\right\}$. It turns out that

$$
K=\left\{k=\left[\begin{array}{cc}
B & 0 \\
0 & 1
\end{array}\right]: B \in S O(n)\right\}
$$

It is well known that $G=S O_{0}(n, 1)$ is a noncompact, connected, rank one semisimple Lie group and $K$ is a maximal compact subgroup of $S O_{0}(n, 1)$ ([27, p.2). It follows that $\mathbb{H}^{n}$ is diffeomorphic to the homogeneous space $S O_{0}(n, 1) / K$ with the identification $g K \mapsto g \cdot x_{0}$. Hence $\mathbb{H}^{n}$ is a Riemannian symmetric space of noncompact type (see [27, p. 2). The $G$-invariant Riemannian metric on $\mathbb{H}^{n}$ is given by

$$
d(x, y)=\cosh ^{-1}([x, y]), \quad x, y \in \mathbb{H}^{n},
$$

([18 Theorem 3.2.2). To proceed further we need the analogs of $d$-dimensional planes in $\mathbb{H}^{n}$. This requires the notion of bispherical coordinates on $\mathbb{H}^{n}$. Bispherical coordinates are natural generalizations of the notion of polar coordinates. For $1 \leq d \leq n$, let $\mathbb{R}^{n+1}=R^{n-d} \oplus R^{d+1}$ where $R^{n-d}=\operatorname{span}\left\{e_{1}, \ldots, e_{n-d}\right\}$ and $R^{d+1}=\operatorname{span}\left\{e_{n-d+1}, \ldots, e_{n+1}\right\}$. The following lemma can be found in [27, page 12 . 
Lemma 3.1. If $1 \leq d \leq n-1$ then every $x \in \mathbb{H}^{n}$, can be written as

$$
x=\zeta \sinh u+\eta \cosh u,
$$

where $0 \leq u<\infty, \zeta \in S^{n-d-1} \subset R^{n-d}$, and $\eta \in \mathbb{H}^{d} \subset R^{d+1}$.

We note that if $x \in \mathbb{H}^{n} \backslash\left(\mathbb{H}^{n} \cap R^{d+1}\right)$ then the above representation is unique. In addition, if we consider the case $d=0$ then it follows that every $x \in \mathbb{H}^{n}, x \neq x_{0}$ has a unique expression

$$
x=\omega \sinh r+e_{n+1} \cosh r
$$

where $\omega \in S^{n-1} \subset R^{n}$. This is the so called polar coordinate representation of the point $x \in \mathbb{H}^{n}$.

The $G$-invariant measure on $\mathbb{H}^{n}$ with respect to the polar coordinates is given by

$$
d x=c_{n} \sinh ^{n-1} r d r d \sigma_{n-1}(\omega) .
$$

Since our main object of study is the analogue of $d$-plane transform, we also need a good understanding of the analogues of $d$-dimensional planes in $\mathbb{H}^{n}$.

Definition 3.2. For $1 \leq d \leq n$ we define $\Lambda_{d}$ to be the set of all linear subspaces $V$ of $\mathbb{R}^{n+1}$ such that: i) $\operatorname{dim} V=d+1$.

ii) There exists $v \in V$ such that $[v, v]>0$.

Since every $V \in \Lambda_{d}$ contains a vector $v$ with $[v, v]>0$, it follows that a suitable scalar multiple of $v$ is in $\mathbb{H}^{n}$ and hence $\mathbb{H}^{n} \cap V \neq \phi$.

Definition 3.3. $\xi \subset \mathbb{H}^{n}$ is called a hyperbolic d-plane (or simply d-plane) if there exists $V \in \Lambda_{d}$ such that $\xi=\mathbb{H}^{n} \cap V$. The set of all d-planes of $\mathbb{H}^{n}$ is denoted by $\Xi_{d}$.

Note that the case $d=1$ describes all the geodesics of $\mathbb{H}^{n}$ ([18, p. 64). The importance of the $d$-planes comes from the fact that they are precisely the totally geodesic submanifolds of $\mathbb{H}^{n}$, that is, the geodesics of these submanifolds are geodesics of $\mathbb{H}^{n}$ (see [18], p. 72).

The set $\Xi_{d}$ can also be thought of as a homogeneous space of the group $G=S O_{0}(n, 1)$ as follows. We fix a particular $d$-plane $\xi_{0} \in \Xi_{d}$ given by $\xi_{0}=\mathbb{H}^{n} \cap \operatorname{span}\left\{e_{n-d+1}, \ldots, e_{n+1}\right\}$. Using Theorem 3.1.6 of [18] it follows that the action of $S O_{0}(n, 1)$ on $\Lambda_{d}$ is transitive and consequently the action of $S O_{0}(n, 1)$ on $\Xi_{d}$ is also transitive. It is not hard to see that the isotropy subgroup at $\xi_{0}$ is isomorphic to $H=S O(n-d) \times S O_{0}(d, 1)$ and hence $\Xi_{d}$ is homeomorphic to $G / H$. It follows from the above discussion that $\xi_{0}$ can be described as

$$
\xi_{0}=\left\{h x_{0}: h \in S O(n-d) \times S O_{0}(d, 1)\right\} .
$$

Since the action of $S O_{0}(n, 1)$ on $\Xi_{d}$ is transitive we have that given any $\xi \in \Xi_{d}$ there exists $g(\xi) \in$ $S O_{0}(n, 1)$ such that $g(\xi) \cdot \xi_{0}=\xi$ (note that $g(\xi)$ is unique modulo $H$ ). So the element of $\Xi_{d}$ are nothing but the $G$-translates of $\xi_{0}$. Let $\mu$ denotes the $G$-invariant measure on the set $\Xi_{d}$. The explicit expression of the measure $\mu$ is given by the following lemma ([2], Lemma 2.1).

Lemma 3.4. If $F$ is a nonnegative measurable function on $\Xi_{d}$ then

$$
\int_{\Xi_{d}} F(\xi) d \mu(\xi)=\int_{0}^{\infty}\left(\int_{K} F\left(k g_{u}^{-1} \xi_{0}\right) d k\right)(\sinh u)^{n-d-1}(\cosh u)^{d} d u,
$$

where $d k$ denotes the normalized Haar measure on $K$ and

$$
g_{u}=\left[\begin{array}{ccc}
\cosh u & 0 & \sinh u \\
0 & I_{n-1} & 0 \\
\sinh u & 0 & \cosh u
\end{array}\right]
$$


We are now in a position to describe the notion of the $d$-dimensional totally geodesic Radon transform. If $f \in C_{c}^{\infty}\left(\mathbb{H}^{n}\right)$ and $\xi \in \Xi_{d}$ then the totally geodesic $d$-dimensional Radon transform (or simply $d$-plane transform) of $f$ at $\xi$ is defined as

$$
R_{d}^{-} f(\xi)=\int_{H} f\left(g(\xi) h . x_{0}\right) d h
$$

where $d h$ is the Haar measure of the group $H$. Since any $\xi$ is of the form $g . \xi_{0}$ for some $g \in S O_{0}(n, 1)$ the above definition is equivalent to

$$
R_{d}^{-} f\left(g \cdot \xi_{0}\right)=\int_{H} f\left(g h \cdot x_{0}\right) d h .
$$

Though we have defined the $d$-plane transform only for $C_{c}^{\infty}$ functions but it turns out that the $d$-plane transform also makes sense for certain class of $L^{p}$ functions. We now quote a result from [2] which gives a precise description of this class.

Theorem 3.5. If $1 \leq p<\frac{n-1}{d-1}$ and $f \in L^{p}\left(\mathbb{H}^{n}\right)$ then the integral in (3.30) converges for almost every $\xi \in \Xi_{d}$. Consequently $R_{d}^{-} f(\xi)$ is well defined for almost every $\xi \in \Xi_{d}$. Moreover if $p \geq \frac{n-1}{d-1}$ then there exists a nonnegative radial function $f$ such that $R_{d}^{-} f(\xi)=\infty$ for almost every $\xi \in \Xi_{d}$.

3.2. The $d$-Plane Transform of Radial Functions on $\mathbb{H}^{n}$. In this section we will concentrate mainly on the class of radial functions. It turns out that, similar to Euclidean spaces, the $d$-plane transform of a radial function on $\mathbb{H}^{n}$ can also be written down explicitly involving an Abel type integral ([2] Lemma 3.1). For the reader's benefit we start with a brief discussion of the $d$-plane transform of radial functions on $\mathbb{H}^{n}$. A function $f$ on $\mathbb{H}^{n}$ is called radial if $f(x)=f(y)$ whenever $d\left(x_{0}, x\right)=d\left(x_{0}, y\right)$. Since the maximal compact subgroup $K$ acts transitively on the unit sphere (see [12]) it follows that a function $f$ is radial if and only if $f(k x)=f(x)$ for all $k \in K$ and $x \in \mathbb{H}^{n}$. We have from (3.23) that $d\left(x_{0}, x\right)=\cosh ^{-1}\left(x_{n+1}\right)$ and hence a radial function $f$ depends only on the last component of $x$, that is, on $x_{n+1}$. Given a radial function $f$ on $\mathbb{H}^{n}$ we define a function $\tilde{f}$ on $[1, \infty)$ by

$$
\tilde{f}(t)=f\left(x_{1}, \ldots, x_{n}, t\right), \quad\left(x_{1}, \ldots, x_{n}, t\right) \in \mathbb{H}^{n} .
$$

The following lemma ( [2], Lemma 3.1) explicitly describes the $d$-plane transform of a radial function on $\mathbb{H}^{n}$.

Lemma 3.6. If $f \in \mathbb{C}_{c}^{\infty}\left(\mathbb{H}^{n}\right)$ is a radial function then for $\xi \in \Xi_{d}$,

$$
\begin{aligned}
R_{d}^{-} f(\xi) & =\frac{C_{d}}{(\cosh s)^{d-1}} \int_{\cosh s}^{\infty} \tilde{f}(t)\left(t^{2}-\cosh ^{2} s\right)^{\frac{d-2}{2}} d t \\
& =\frac{C_{d}}{\cosh s} \int_{s}^{\infty} \tilde{f}(\cosh r)\left(1-\frac{\tanh ^{2} s}{\tanh ^{2} r}\right)^{\frac{d-2}{2}} \sinh ^{d-1} r d r
\end{aligned}
$$

where $s=d\left(x_{0}, \xi\right)$.

For a radial function $f$ on $\mathbb{H}^{n}$ we define the Abel transform of $f$ by $A_{d}^{-} f(s)=R_{d}^{-} f(\xi)$ where $s=d\left(x_{0}, \xi\right)$. It is important for us to get an explicit expression of the $L^{p}$ norm of $A_{d}^{-} f$. In this regard, we first observe that for $k \in K$,

$$
d\left(x_{0}, k g_{u}^{-1} \xi_{0}\right)=d\left(g_{u} k^{-1} x_{0}, \xi_{0}\right)=d\left(g_{u} x_{0}, \xi_{0}\right)=\inf _{y \in \xi_{0}} d\left(g_{u} x_{0}, y\right)=d\left(g_{u} x_{0}, x_{0}\right)=u .
$$


It follows that for radial functions $R_{d}^{-} f\left(k g_{u}^{-1} \xi_{0}\right)=A_{d}^{-} f(u)$. It now follows from Lemma 3.4 that if $f$ is radial function on $\mathbb{H}^{n}$ then for $1 \leq p<\infty$,

$$
\int_{\Xi_{d}}\left|R_{d}^{-} f(\xi)\right|^{p} d \mu(\xi)=C_{n, d} \int_{0}^{\infty}\left|A_{d}^{-} f(u)\right|^{p}(\sinh u)^{n-d-1}(\cosh u)^{d} d u .
$$

Using (3.35) and (3.26) it is easy to see that ([2], Corollary 2.4),

$$
\left\|A_{d}^{-} f\right\|_{L^{1}\left(\Xi_{d}\right)} \leq C\|f\|_{L^{1}\left(\mathbb{H}^{n}\right)} .
$$

We will now prove a lemma which seems to be a recurring theme as far as $L^{p}-L^{q}$ mapping property of Abel transforms is concerned. The following lemma is implicit in the proof of (1.5) as well as in the proof of Theorem 2.2. A special case of the lemma also played a crucial role in the end-point estimate of the horospherical Radon transform of radial functions on rank one symmetric spaces of noncompact type ([13], Lemma 3). Though we are interested in the totally geodesic $d$-dimensional Radon transform (instead of the horospherical Radon transform) of radial functions on $\mathbb{H}^{n}$ and $S^{n}$, it turns out that this lemma is still an essential ingredient for the results to follow.

Lemma 3.7. If $\delta \neq 0$ then there exists a positive constant $C_{\delta}$ such that for all indicator functions of measurable subsets of $\mathbb{R}$ the following inequality holds,

$$
\int_{\mathbb{R}} f(t) e^{\delta t} d t \leq C_{\delta}\left(\int_{\mathbb{R}} f(t) e^{p \delta t} d t\right)^{1 / p} .
$$

Proof. Using the change of variable $e^{\delta t}=s$ it suffices to prove the inequality

$$
\int_{0}^{\infty} \phi(s) d s \leq C_{\delta}\left(\int_{0}^{\infty} \phi(s) s^{p-1} d s\right)^{1 / p},
$$

for all indicator functions $\phi$ of measurable subsets of $(0, \infty)$. We first consider the case when $\phi$ is of the form $\chi_{\cup_{i=1}^{k}\left[a_{i}, b_{i}\right]}$ with $a_{1}<b_{1} \leq a_{2}<b_{2} \leq \cdots \leq a_{k}<b_{k}$. For functions of this form (3.38) follows immediately from (2.10) by considering $x_{1}=b_{k}, x_{2}=a_{k}, \ldots, x_{2 k-1}=b_{1}, x_{2 k}=a_{1}$. Since every nonempty open subset of $\mathbb{R}$ is a countable disjoint union of open intervals, the result holds for indicator function of open sets by monotone convergence theorem. Now suppose that $E$ is a measurable subset of $(0, \infty)$. By monotone convergence theorem it suffices to prove the result for $E \cap(0, m)$ for each $m \in \mathbb{N}$. So without loss of generality we can assume that $E \subset(0, \lambda)$ for some $\lambda>0$. By regularity of the Lebesgue measure we can get a decreasing family of open sets $\left\{U_{n}\right\}$ such that $E \subset U_{n} \subset(0, \lambda)$ for each $n$ and $\left|U_{n} \backslash E\right|<\frac{1}{n}$ (here $|E|$ denotes Lebesgue measure of the set $E$ ). So

$$
|E|=\left|U_{n}\right|-\left|U_{n} \backslash E\right| \leq\left(\int_{0}^{\infty} \chi_{U_{n}}(s) s^{p-1} d s\right)^{1 / p}-\left|U_{n} \backslash E\right| .
$$

It now suffices to show that

$$
\lim _{n \rightarrow \infty} \int_{0}^{\infty} \chi_{U_{n}}(s) s^{p-1} d s=\int_{0}^{\infty} \chi_{E}(s) s^{p-1} d s .
$$

If $d \nu(s)=s^{p-1} d s$ then

$$
\begin{aligned}
& \lim _{n \rightarrow \infty} \int_{0}^{\infty} \chi_{U_{n}}(s) s^{p-1} d s-\int_{0}^{\infty} \chi_{E}(s) s^{p-1} d s \\
= & \lim _{n \rightarrow \infty} \int_{0}^{\infty} \chi_{U_{n} \backslash E}(s) s^{p-1} d s \\
= & \nu\left(\cap_{n=1}^{\infty}\left(U_{n} \backslash E\right)\right) .
\end{aligned}
$$


Since Lebesgue measure of the set $\cap_{n=1}^{\infty}\left(U_{n} \backslash E\right)$ is zero it follows that

$$
\lim _{n \rightarrow \infty} \int_{0}^{\infty} \chi_{U_{n} \backslash E}(s) s^{p-1} d s=0 .
$$

Remark 3.8. Using the substitution $t=\operatorname{logs}$ it follows from (3.37) that

$$
\int_{0}^{\infty} \phi(s) s^{\delta-1} d s \leq C_{\delta}\left(\int_{0}^{\infty} \phi(s) s^{p \delta-1} d s\right)^{1 / p}
$$

We are now in a position to state and prove the main result of this section.

Theorem 3.9. a) If $n \geq 3$ and $2 \leq d \leq n-1$ then there exists a constant $C>0$ such that for all measurable, radial functions $f$ on $\mathbb{H}^{n}$ the following inequality holds,

$$
\left\|A_{d}^{-} f\right\|_{L^{\infty}\left(\Xi_{d}\right)} \leq C\|f\|_{L^{\frac{n-1}{d-1}, 1}\left(\mathbb{H}^{n}\right)} .
$$

b) If $n \geq 2$ and $1 \leq d \leq n-1$ then there exists a constant $C>0$ such that for all measurable radial functions on $\mathbb{H}^{n}$ the following inequality holds,

$$
\left\|A_{d}^{-} f\right\|_{L^{q}\left(\Xi_{d}\right)} \leq C\|f\|_{L^{p}\left(\mathbb{H}^{n}\right)},
$$

where

$$
\frac{n-1}{p}=d-1+\frac{n-d}{q}, \quad 1 \leq p<\frac{n-1}{d-1}
$$

Proof. We will first prove part $a$ ) of the theorem. As in Theorem 2.2 it is enough to prove the result for indicator functions of all measurable, radial subsets of $\mathbb{H}^{n}$ (see [26], Theorem 3.13 ). We will show that there exists a constant $C>0$ such that

$$
\left|\cosh (s) A_{d}^{-} f(s)\right| \leq C\left(\int_{\mathbb{H}^{n}} f(x) d x\right)^{\frac{d-1}{n-1}}=C\left(\int_{0}^{\infty} \tilde{f}(\cosh t) \sinh ^{n-1} t d t\right)^{\frac{d-1}{n-1}},
$$

where $s \in(0, \infty)$ and $f$ is indicator function of a radial, measurable subset of $\mathbb{H}^{n}$. Using (3.34) we write

$$
\cosh (s) A_{d}^{-} f(s)=\sigma_{d-1} \int_{s}^{\infty} \tilde{f}(\cosh t)\left(1-\frac{\tanh ^{2} s}{\tanh ^{2} t}\right)^{\frac{d-2}{2}} \sinh ^{d-1} t d t=I_{1}+I_{2}
$$

where

$$
\begin{aligned}
& I_{1}=\sigma_{d-1} \int_{s}^{s+\alpha} \tilde{f}(\cosh t)\left(1-\frac{\tanh ^{2} s}{\tanh ^{2} t}\right)^{\frac{d-2}{2}} \sinh ^{d-1} t d t, \\
& I_{2}=\sigma_{d-1} \int_{s+\alpha}^{\infty} \tilde{f}(\cosh t)\left(1-\frac{\tanh ^{2} s}{\tanh ^{2} t}\right)^{\frac{d-2}{2}} \sinh ^{d-1} t d t,
\end{aligned}
$$

and $\alpha=1 / 2$. We will estimate $I_{1}$ and $I_{2}$ separately. For $t \in(s+\alpha, \infty)$ we have $\tanh s<\tanh t$ and $\sinh t \asymp e^{t}$. So

$$
I_{2} \leq C \int_{s+\alpha}^{\infty} \tilde{f}(\cosh t) e^{(d-1) t} d t
$$


We now appeal to Lemma 3.7 with $\delta=d-1$ and $p=\frac{n-1}{d-1}$ to get

$$
\begin{aligned}
\int_{s+\alpha}^{\infty} \tilde{f}(\cosh t) e^{(d-1) t} d t & \leq C\left(\int_{s+\alpha}^{\infty} \tilde{f}(\cosh t) e^{(n-1) t} d t\right)^{\frac{d-1}{n-1}} \\
& \asymp C\left(\int_{s+\alpha}^{\infty} \tilde{f}(\cosh t) \sinh ^{n-1} t d t\right)^{\frac{d-1}{n-1}} \\
& \leq C\|f\|_{L^{\frac{n-1}{d-1}, 1}\left(\mathbb{H}^{n}\right)} .
\end{aligned}
$$

By similar argument as above we can prove the required estimate for $I_{1}$ if $s \geq \alpha$. We will now estimate $I_{1}$ when $0<s<\alpha$. We first observe that by mean value theorem that there exists a real number $u \in(s, t)$ such that

$$
\begin{aligned}
& \int_{s}^{s+\alpha} \tilde{f}(\cosh t)\left(\tanh ^{2} t-\tanh ^{2} s\right)^{\frac{d-2}{2}} \operatorname{coth}^{d-2} t \sinh ^{d-1} t d t \\
& =\int_{s}^{s+\alpha} \tilde{f}(\cosh t)(t-s)^{\frac{d-2}{2}}\left(\frac{2 \tanh u}{\cosh ^{2} u}\right)^{\frac{d-2}{2}} \frac{\sinh ^{d-1} t}{\tanh ^{d-2} t} d t
\end{aligned}
$$

If $0<r<1$ then we have $\sinh r \asymp r$ and $\cosh r \asymp 1$. As $s<u<t<s+\alpha<1$ and $d \geq 2$ it follows from the Remark 3.8 and (3.49) that

$$
\begin{aligned}
I_{1} & \leq C \int_{s}^{s+\alpha} \tilde{f}(\cosh t)(t-s)^{\frac{d-2}{2}}(u)^{\frac{d-2}{2}} t d t \\
& \leq C \int_{s}^{s+\alpha} \tilde{f}(\cosh t)(t-s)^{\frac{d-2}{2}} t^{\frac{d}{2}} d t \\
& \leq C \int_{s}^{s+\alpha}\left(\tilde{f}(\cosh t) t^{d-1}\right)\left((t-s)^{\frac{d-2}{2}} t^{\frac{2-d}{2}}\right) d t \\
& \leq C \int_{s}^{s+\alpha} \tilde{f}(\cosh t) t^{d-1} d t \\
& \leq C \int_{s}^{s+\alpha} \tilde{f}(\cosh t) t^{n(d-1) /(n-1)-1} d t \\
& \leq C\left(\int_{s}^{s+\alpha} \tilde{f}(\cosh t) t^{n-1} d t\right)^{\frac{d-1}{n-1}} \\
& \leq C\left(\int_{s}^{s+\alpha} \tilde{f}(\cosh t) \sinh ^{n-1} t d t\right)^{\frac{d-1}{n-1}} \\
& \leq C\|f\|_{L^{\frac{n-1}{d-1}, 1}\left(\mathbb{H}^{n}\right)}
\end{aligned}
$$

This proves (3.44). As $\cosh s \geq 1$ this completes the proof of $a$ ).

The proof of $b$ ), for $2 \leq d \leq n-1$, follows by interpolating ([6], Theorem 1.4.19) between the estimates (3.41) and (3.36).

For $d=1$, the relation (3.43) shows that we need to prove the inequality

$$
\left\|A_{1}^{-} f\right\|_{L^{p}\left(\Xi_{d}\right)} \leq C\|f\|_{L^{p}\left(\mathbb{H}^{n}\right)}, \quad 1 \leq p<\infty .
$$

We first write the formula for $A_{1}^{-} f$ given by (3.33) as follows

$$
A_{1}^{-} f(s)=C \int_{\cosh s}^{\infty} \tilde{f}(t)\left(t^{2}-\cosh ^{2} s\right)^{-\frac{1}{2}} d t .
$$


From (3.35) we have

$$
\begin{gathered}
\left\|A_{1}^{-} f\right\|_{L^{p}\left(\Xi_{1}\right)}=C\left(\int_{0}^{\infty}\left|A_{1}^{-} f(s)\right|^{p} \sinh ^{n-2} s \cosh s d s\right)^{1 / p} \\
=C\left(\int_{0}^{\infty}\left(\int_{\cosh s}^{\infty} \tilde{f}(t)\left(t^{2}-\cosh ^{2} s\right)^{-\frac{1}{2}} d t\right)^{p} \sinh ^{n-2} s \cosh s d s\right)^{1 / p},
\end{gathered}
$$

(see Lemma 3.6). We note that $\tilde{f}$ is a positive function. Using the substitution $\cosh s=r$, we get

$$
\left\|A_{1}^{-} f\right\|_{L^{p}\left(\Xi_{1}\right)}=C\left(\int_{1}^{\infty}\left(\int_{r}^{\infty} \tilde{f}(t)\left(t^{2}-r^{2}\right)^{-\frac{1}{2}} d t\right)^{p}\left(r^{2}-1\right)^{\frac{n-3}{2}} r d r\right)^{1 / p}
$$

Again, using the change of variables $t^{2}=v+1, r^{2}=u+1$, we get

$$
\begin{aligned}
\left\|A_{1}^{-} f\right\|_{L^{p}\left(\Xi_{1}\right)}= & C\left(\int_{0}^{\infty}\left(\int_{u}^{\infty} \tilde{f}(\sqrt{v+1})(v-u)^{-\frac{1}{2}}(v+1)^{-\frac{1}{2}} d v\right)^{p} u^{\frac{n-3}{2}} d u\right)^{1 / p} \\
= & C\left(\int _ { 0 } ^ { \infty } \left(\int_{0}^{\infty} \tilde{f}(\sqrt{v+1})(v+1)^{-\frac{1}{2 p}} v^{\frac{n}{2 p}}\left(\frac{v}{1+v}\right)^{\frac{1}{2 p^{\prime}}}\right.\right. \\
& \left.\left.\times\left(1-\frac{u}{v}\right)^{-\frac{1}{2}}(u / v)^{\frac{n-1}{2 p}} \chi_{[0,1]}(u / v) \frac{d v}{v}\right)^{p} \frac{d u}{u}\right)^{1 / p} \\
\leq & C\left(\int _ { 0 } ^ { \infty } \left(\int_{0}^{\infty} \tilde{f}(\sqrt{v+1})(v+1)^{-\frac{1}{2 p}} v^{\frac{n}{2 p}}\right.\right. \\
& \left.\left.\times\left(1-\frac{u}{v}\right)^{-\frac{1}{2}}(u / v)^{\frac{n-1}{2 p}} \chi_{[0,1]}(u / v) \frac{d v}{v}\right)^{p} \frac{d u}{u}\right)^{1 / p}, \quad\left(\text { as } \frac{v}{1+v}<1\right) .
\end{aligned}
$$

By Young's inequality for the group $(0, \infty)$, we get,

$$
\begin{aligned}
\left\|A_{1}^{-} f\right\|_{L^{p}\left(\Xi_{1}\right)} \leq & C\left(\int_{0}^{\infty}(\tilde{f}(\sqrt{v+1}))^{p}(v+1)^{-\frac{1}{2}} v^{\frac{n}{2}} \frac{d v}{v}\right)^{1 / p} \\
& \times\left(\int_{0}^{\infty}(1-u)^{-\frac{1}{2}} u^{\frac{n-1}{2 p}} \chi_{[0,1]}(u) \frac{d u}{u}\right) \\
\leq & C\left(\int_{1}^{\infty}(\tilde{f}(s))^{p}\left(s^{2}-1\right)^{\frac{n-2}{2}} d s\right)^{1 / p} \\
& \quad\left(\text { by using } v+1=s^{2}\right) \\
\leq & C\left(\int_{0}^{\infty}(\tilde{f}(\cosh r))^{p} \sinh ^{n-1} r d r\right)^{1 / p} \\
& \quad(\text { by using } s=\cosh r) \\
= & C\|f\|_{L^{p}\left(\mathbb{H}^{n}\right)}
\end{aligned}
$$

This completes the proof.

Remark 3.10. Though Theorem 3.9 is analogous to the corresponding result on Euclidean space ([5], Theorem 1) but it does not seem to reveal the full story. It turns out that the $L^{p}-L^{q}$ mapping property of the Abel transform on $\mathbb{H}^{n}$ is very different from that of Euclidean space. In this regard, we observe that if $d \geq 2$ then (3.44) shows that if $f \in L^{\frac{n-1}{d-1}, 1}\left(\mathbb{H}^{n}\right)$ then

$$
\left|A_{d}^{-} f(s)\right| \leq C_{f}(\cosh s)^{-1}, \quad s>0,
$$


where $C_{f}$ is a constant multiple of $\|f\|_{L^{\frac{n-1}{d-1}, 1}\left(\mathbb{H}^{n}\right)}$. Since the function $g(s)=1 / \cosh s$ decays like $e^{-s}$ at infinity and the $G$-invariant measure on $\Xi_{d}$ grows like $e^{(n-1) s}$ at infinity (see Lemma 3.4) it follows that $g \in L^{n-1, \infty}\left(\Xi_{d}, \mu\right)$. As a consequence, we have

$$
\left\|A_{d}^{-} f\right\|_{L^{n-1, \infty}\left(\Xi_{d}\right)} \leq C\|f\|_{L^{\frac{n-1}{d-1}, 1}\left(\mathbb{H}^{n}\right)} .
$$

Corollary 3.11. If $2 \leq d \leq n-1,1 \leq p<\frac{n-1}{d-1}$ and $\frac{n-\kappa}{p}=(d-\kappa)+\frac{n-d}{q_{\kappa}}$, then for all $\kappa \in[1,2]$ the following inequality holds,

$$
\left\|A_{d}^{-} f\right\|_{q_{\kappa}\left(\Xi_{d}\right)} \leq C_{\kappa}\|f\|_{L^{p}\left(\mathbb{H}^{n}\right)},
$$

for all radial measurable function on $\mathbb{H}^{n}$.

Proof. From (3.43) we have

$$
\begin{gathered}
\left\|A_{d}^{-} f\right\|_{L^{q}\left(\Xi_{d}\right)} \leq C\|f\|_{L^{p}\left(\mathbb{H}^{n}\right)}, \\
\frac{n-1}{p}=d-1+\frac{n-d}{q}, \quad 1 \leq p<\frac{n-1}{d-1} .
\end{gathered}
$$

By interpolating between the estimates (3.36) and (3.53) we get

$$
\begin{gathered}
\left\|A_{d}^{-} f\right\|_{L^{q}\left(\Xi_{d}\right)} \leq C\|f\|_{L^{p}\left(\mathbb{H}^{n}\right)}, \\
\frac{n-2}{p}=d-2+\frac{n-d}{q}, \quad 1 \leq p<\frac{n-1}{d-1} .
\end{gathered}
$$

If we fix $p \in\left(1, \frac{n-1}{d-1}\right)$ then there exist $q_{1}$ and $q_{2}$ such that

$$
\left\|A_{d}^{-} f\right\|_{L^{q_{j}}\left(\Xi_{d}\right)} \leq C\|f\|_{L^{p}\left(\mathbb{H}^{n}\right)}, \quad j=1,2,
$$

where $q_{1}$ and $q_{2}$ are given by (3.56) and (3.58) respectively. It is easy to see from above that $q_{2}<q_{1}$. Hence elements of $\left[q_{2}, q_{1}\right]$ can be written as

$$
\frac{1}{q_{\kappa}}=\frac{2-\kappa}{q_{1}}+\frac{\kappa-1}{q_{2}}, \quad \kappa \in[1,2] .
$$

It is now easy to see that $q_{\kappa}$ satisfies the relation,

$$
\frac{n-\kappa}{p}=(d-\kappa)+\frac{n-d}{q_{\kappa}} .
$$

Since $q_{2}<q_{\kappa}<q_{1}$ we have (see [6], Proposition 1.1.14)

$$
\left\|A_{d}^{-} f\right\|_{L^{q_{\kappa}}\left(\Xi_{d}\right)} \leq C_{p, q_{1}, q_{2}}\left\|A_{d}^{-} f\right\|_{L^{q_{2}}\left(\Xi_{d}\right)}^{\frac{\frac{1}{q_{\kappa}}-\frac{1}{q_{1}}}{q_{2}}}\left\|A_{d}^{-} f\right\|_{L^{q_{1}}\left(\Xi_{d}\right)}^{\frac{\frac{1}{q_{2}}-\frac{1}{q_{\kappa}}}{q_{2}}-\frac{1}{q_{1}}} .
$$

The result now follows by applying (3.55) and (3.57).

\section{The Sphere}

4.1. Notation and Preliminaries. In this section we will discuss about the notion of the $d$-dimensional totally geodesic Radon transform on the unit sphere. Since the situation here is analogous 
to that of $\mathbb{H}^{n}$ our exposition will be brief. We start with a few notation. Let

$$
\begin{aligned}
S^{n} & =\left\{x=\left(x_{1}, \ldots, x_{n+1}\right) \in \mathbb{R}^{n+1}: \sum_{i=1}^{n+1} x_{i}^{2}=1\right\}, \\
R^{n-d} & =\operatorname{span}\left\{e_{1}, \ldots, e_{n-d}\right\}, \\
R^{d+1} & =\operatorname{span}\left\{e_{n-d+1}, \ldots, e_{n+1}\right\}, \\
\xi_{0} & =R^{d+1} \cap S^{n}=S^{d}, x_{0}=e_{n+1},
\end{aligned}
$$

where $\left\{e_{1}, \ldots, e_{n+1}\right\}$ is the standard orthonormal basis of $\mathbb{R}^{n}$ and $1 \leq d \leq n-1$. We note that the situation here is little different from that of $\mathbb{H}^{n}$, in the sense that $x \in \xi_{0}$ if and only if $-x \in \xi_{0}$. The compact Lie group $G=S O(n+1)$ acts transitively on $S^{n}$ and the isotropy subgroup at $x_{0}$ is given by

$$
K=\left\{\left(\begin{array}{ll}
k & 0 \\
0 & 1
\end{array}\right): k \in S O(n)\right\} \approx S O(n) .
$$

Hence $S^{n}$ can be viewed as the homogeneous space $G / K$.

It is known that all $d$-dimensional totally geodesic submanifolds of $S^{n}$ are intersections of $S^{n}$ with $(d+1)$-dimensional subspaces of $\mathbb{R}^{n+1}([18]$, p. 40). Hence the set of $d$-dimensional totally geodesic submanifolds of $S^{n}$ can be parametrized by $G_{n+1, d+1}$. We note that $\xi_{0} \in G_{n+1, d+1}$ and for every $\xi \in G_{n+1, d+1}, x \in \xi$ if and only if $-x \in \xi$. The group $G$ also acts transitively on $G_{n+1, d+1}$ with the isotropy subgroup at $\xi_{0}$ given by

$$
H=\left\{h=\left(\begin{array}{cc}
S & 0 \\
0 & T
\end{array}\right): T \in S O(d+1), S \in S O(n-d)\right\} \approx S O(n-d) \times S O(d+1) .
$$

Thus $G_{n+1, d+1}$ is also a homogeneous space of the group $S O(n+1)$, namely, $G_{n+1, d+1} \approx G / H([20$, p. 78). In view of the above discussion it is now easy to see that if $\xi \in G_{n+1, d+1}$ then there exists a $g(\xi) \in S O(n)$ (which is unique modulo $H$ ) such that

$$
\xi_{0}=\left\{h x_{0}: h \in H\right\}, \quad \xi=g(\xi) \xi_{0}=\left\{g(\xi) h x_{0}: h \in H\right\} .
$$

We are now in a position to define the notion of $d$-dimensional totally geodesic Radon transform $(d$-plane transform) on the sphere.

Definition 4.1. Given a continuous function $f$ defined on $S^{n}$ we define the d-dimensional totally geodesic Radon transform of $f$ as

$$
R_{d}^{+} f(\xi)=\int_{H} f\left(g(\xi) h x_{0}\right) d h, \quad \xi \in G_{n+1, d+1}
$$

where dh stands for the normalized Haar measure on the compact group $H$.

Using the identification of $\xi_{0}$ with $S^{d}$ one can see that (4.59) can also be written as

$$
R_{d}^{+} f(\xi)=\int_{S^{d}} f(g(\xi) y) d \sigma_{d}(y)
$$

Remark 4.2. We note the following important difference between $d$-plane transform on $\mathbb{H}^{n}$ and $S^{n}$. As $\xi$ is invariant under reflection about the origin it follows from (4.59) that $R_{d}^{+} f(\xi)=0$ for all $\xi \in G_{n+1, d+1}$ if $f$ is an odd function.

We will now specialize to the class of radial functions on $S^{n}$. An explicit formula for $d$-plane transform of radial functions appear in [20] (see also [11]). For the sake of completeness we explain it in some detail. 
Definition 4.3. A function $f$ defined on $S^{n}$ is called radial (or zonal) if for all $x \in S^{n}$ and for all $k \in K, f$ satisfies the condition $f(k x)=f(x)$.

To understand the radial functions we use the notion of polar coordinate on the sphere. Every element $x \in S^{n}$, with $x \neq x_{0}$ can be uniquely written as

$$
x=\rho \sin \theta+x_{0} \cos \theta, \quad \rho \in S^{n-1}, 0<\theta \leq \pi,
$$

or equivalently $x=k$. $\left(\sin \theta e_{1}+x_{0} \cos \theta\right), \quad k \in K$. For radial functions $f$ it now follows that $f(x)=$ $f\left(\rho \sin \theta+x_{0} \cos \theta\right)=f\left(\sin \theta e_{1}+x_{0} \cos \theta\right)$. Hence a radial functions $f$ on $S^{n}$ can be thought of as a function $\tilde{f}$ on the interval $[0, \pi]$ given by the relation

$$
\tilde{f}(\cos \theta)=f(x)=f\left(\rho \sin \theta+x_{0} \cos \theta\right) .
$$

The Riemannian metric on $S^{n}$ is given by $d_{2}(x, y)=\cos ^{-1}(\langle x, y\rangle)$, ([18, p. 36). Since $d_{2}(\rho \sin \theta+$ $\left.x_{0} \cos \theta, x_{0}\right)=\theta$, it follows that a radial function on $S^{n}$ is actually a function of the distance of a point from $x_{0}$. To proceed further we need the notion of bispherical coordinate on $S^{n}$ ([27], p. 23). If $1 \leq d \leq n-1$ then every $x \in S^{n}$ can be written as

$$
x=\eta \cos \theta+\zeta \sin \theta,
$$

where $\eta \in S^{d}=R^{d+1} \cap S^{n}, \zeta \in S^{n-d-1}=R^{n-d} \cap S^{n}$, and $\theta \in\left[0, \frac{\pi}{2}\right]$. In these coordinates the $G$ invariant measure on $S^{n}$ is given by

$$
d x=\sin ^{n-d-1} \theta \cos ^{d} \theta d \theta d \eta d \zeta,
$$

where $d \eta$ and $d \zeta$ denote the normalized rotation invariant measures on $S^{d}$ and $S^{n-d-1}$ respectively (27],p. 12, 22). Now, suppose that $f$ is a radial $C^{\infty}$ function on $S^{n}$. Then for $\xi \in G_{n+1, d+1}$ we have from (4.60)

$$
\begin{aligned}
R_{d}^{+} f(\xi) & =\int_{S^{d}} f(g(\xi) y) d \sigma_{d}(y) \\
& =\int_{S^{d}} \tilde{f}\left(\left\langle g(\xi) y, x_{0}\right\rangle\right) d \sigma_{d}(y) \\
& =\int_{S^{d}} \tilde{f}\left(\left\langle y, g(\xi)^{-1} x_{0}\right\rangle\right) d \sigma_{d}(y) \\
& =\int_{S^{d}} \tilde{f}(\langle y, \eta \cos \theta\rangle) d \sigma_{d}(y) .
\end{aligned}
$$

In the last step we have used the bispherical representation $g(\xi)^{-1} x_{0}=\eta \cos \theta+\zeta \sin \theta$ (see (4.63)). To make the above formula more explicit we will need the catalan formula which is described below. let $\psi$ be a function defined on $\mathbb{R}$ and let $x \in \mathbb{R}^{d}, d \geq 2$. Then

$$
\int_{S^{d}} \psi(\langle x, \omega\rangle) d \sigma \omega=C_{d} \int_{-1}^{1} \psi(s\|x\|)\left(1-s^{2}\right)^{\frac{d-2}{2}} d s
$$


For proof of this formula we refer the reader to [6], D.3. Thus

$$
\begin{aligned}
R_{d}^{+} f(\xi) & =\int_{S^{d}} \tilde{f}(\cos \theta\langle y, \eta\rangle) d \sigma_{d}(y) \\
& =C \int_{-1}^{1} \tilde{f}(\cos \theta s)\left(1-s^{2}\right)^{\frac{d-2}{2}} d s \quad \text { (by using (4.65)) } \\
& \left.=C \int_{-\cos \theta}^{\cos \theta} \frac{\tilde{f}(u)}{\cos \theta}\left(1-\frac{u^{2}}{\cos ^{2} \theta}\right)^{\frac{d-2}{2}} d u \quad \text { (using } s \cos \theta=u\right) \\
& =\frac{C}{(\cos \theta)^{d-1}} \int_{-\cos \theta}^{\cos \theta} \tilde{f}(u)\left(\cos ^{2} \theta-u^{2}\right)^{\frac{d-2}{2}} d u .
\end{aligned}
$$

It follows from (4.66) that for radial functions $R_{d}^{+} f$ is a function of $\theta$ only.

In the following the $d$-plane transform of radial functions $f$ will be denoted by $A_{d}^{+} f$ and will be called the Abel transform of $f$.

Lemma 4.4. If $f \in C^{\infty}\left(S^{n}\right)$ is an even, radial function then for $\theta \in\left[0, \frac{\pi}{2}\right]$,

$$
\begin{aligned}
A_{d}^{+} f(\theta) & =\frac{C}{(\cos \theta)^{d-1}} \int_{0}^{\cos \theta} \tilde{f}(u)\left(\cos ^{2} \theta-u^{2}\right)^{\frac{d-2}{2}} d u, \\
& =\frac{C}{\cos \theta} \int_{\theta}^{\pi / 2} \tilde{f}(\cos r)\left(1-\frac{\tan ^{2} \theta}{\tan ^{2} r}\right)^{\frac{d-2}{2}} \sin ^{d-1} r d r .
\end{aligned}
$$

Proof. The first identity follows from (4.66) by using the fact that $f$ is even. The second identity follows from the first one by using the change of variable $u=\cos r$.

We end this section by quoting the following result from [20] regarding the mapping property of $R_{d}^{+}$.

Theorem 4.5. If $1 \leq p \leq \infty$ and $1 \leq d \leq n-1$ then for all continuous functions $f$ on $S^{n}$,

$$
\left\|R_{d}^{+} f\right\|_{L^{p}\left(G_{n+1, d+1}\right)} \leq C\|f\|_{L^{p}\left(S^{n}\right)} .
$$

4.2. The $d$-Plane Transform of Radial Functions on the Sphere. As in the previous section we are interested in the inequalities of the form

$$
\left\|A_{d}^{+} f\right\|_{L^{q}\left(G_{n+1, d+1}\right)} \leq C\|f\|_{L^{p}\left(S^{n}\right)},
$$

which should be valid for all $f \in C^{\infty}\left(S^{n}\right)$. Any $f \in C^{\infty}\left(S^{n}\right)$ can be written as $f=f_{1}+f_{2}$ where $f_{1}(x)=(f(x)+f(-x)) / 2$ is an even function and $f_{2}$ is an odd function. Consequently $A_{d}^{+} f=A_{d}^{+} f_{1}$ (see Remark 4.2). To prove an inequality of the above form it thus suffices to prove an inequality of the form $\left\|A_{d}^{+} f_{1}\right\|_{L^{q}\left(G_{n+1, d+1}\right)} \leq C\left\|f_{1}\right\|_{L^{p}\left(S^{n}\right)}$, as

$$
\left\|A_{d}^{+} f\right\|_{L^{q}\left(G_{n+1, d+1}\right)}=\left\|A_{d}^{+} f_{1}\right\|_{L^{q}\left(G_{n+1, d+1}\right)} \leq C\left\|f_{1}\right\|_{L^{p}\left(S^{n}\right)} \leq C\|f\|_{L^{p}\left(S^{n}\right)} .
$$

So, from now onwards, we will deal only with nonnegative, even functions on $S^{n}$. We start with an example to show that, situation here is different from that of $\mathbb{R}^{n}$ and $\mathbb{H}^{n}$.

Example 4.6. We will show that $A_{d}^{+}$is not bounded from $L^{p, 1}\left(S^{n}\right)$ to $L^{\infty}\left(G_{n+1, d+1}\right)$ if $p<\infty$. Let

$$
f_{i}(s)=\chi_{\left[0, a_{i}\right]}(s), \quad 0<a_{i}<1,
$$


and $\left\{a_{i}\right\}$ be a decreasing sequence converging to 0 . If $a_{i}>\cos s$ then from (4.67) we get

$$
A_{d}^{+} f_{i}(s)=C(\cos s)^{1-d} \int_{0}^{\cos s}\left(\cos ^{2} s-t^{2}\right)^{\frac{d-2}{2}} d t .
$$

It follows from (4.70) that the sequence $\left\{\left\|A_{d}^{+} f_{i}\right\|_{L^{\infty}\left(G_{n+1, d+1}\right)}\right\}$ is bounded away from zero. On the other hand,

$$
\begin{aligned}
\left\|\chi_{\left[0, a_{i}\right]}\right\|_{L^{p, 1}\left(S^{n}\right)} & =C\left(\int_{0}^{\pi / 2} \chi_{\left[0, a_{i}\right]}(\cos s) \sin ^{n-1} s d s\right)^{1 / p} \\
& =C\left(\int_{0}^{1} \chi_{\left[0, a_{i}\right]}(t)\left(1-t^{2}\right)^{\frac{n-2}{2}} d t\right)^{1 / p} \\
& \leq C\left(\int_{0}^{1} \chi_{\left[0, a_{i}\right]}(t)(1-t)^{\frac{n-2}{2}} d t\right)^{1 / p} \\
& =C\left(1-\left(1-a_{i}\right)^{n / 2}\right)^{1 / p} .
\end{aligned}
$$

From (4.71) it is clear that the sequence $\left\{\left\|\chi_{\left[0, a_{i}\right]}\right\|_{L^{p, 1}\left(S^{n}\right)}\right\}_{i=1}^{\infty}$ converges to 0. This implies that Abel transform cannot be bounded from $L^{p, 1}\left(S^{n}\right)$ to $L^{\infty}\left(G_{n+1, d+1}\right)$ if $p<\infty$.

The following theorem can be considered as an analogue of (3.44).

Theorem 4.7. If $1 \leq d \leq n-1$ then for all non-negative $K$ invariant function $f$ on $S^{n}$, there exists a positive constant $C$ such that

$$
\left\|\cos (\cdot) A_{d}^{+} f(\cdot)\right\|_{L^{\infty}\left(G_{n+1, d+1}\right)} \leq C\|f\|_{L^{\frac{n}{d}, 1}\left(S^{n}\right)} .
$$

Proof. As in Theorem 3.9, it is sufficient to prove the result for functions of the form $f(t)=\chi_{E}(t)$, where $E=\bigcup_{i=1}^{m}\left[a_{i}, b_{i}\right], m \in \mathbb{N}$ and $0 \leq a_{1} \leq b_{1} \leq a_{2} \leq \cdots \leq a_{l} \leq b_{l} \leq 1$. We will first prove the result for the case $2 \leq d \leq n-1$. Using (4.68) we write

$$
\cos s A_{d}^{+} f(s)=C \int_{s}^{\pi / 2} f(\cos r)\left(1-\frac{\tan ^{2} s}{\tan ^{2} r}\right)^{\frac{d-2}{2}} \sin ^{d-1} r d r .
$$

Since $s \leq r$, we have $0 \leq 1-\frac{\tan ^{2} s}{\tan ^{2} r} \leq 1$. Now from the above expression we have

$$
\begin{aligned}
\cos s A_{d}^{+} f(s) & \leq C \int_{s}^{\pi / 2} f(\cos r) \sin ^{d-1} r d r \\
& \leq C \int_{0}^{\pi / 2} f(\cos r) r^{d-1} d r
\end{aligned}
$$

By Lemma 3.7 (see also Remark 3.8) we get

$$
\begin{aligned}
\cos s A_{d}^{+} f(s) & \leq C\left(\int_{0}^{\pi / 2} f(\cos r) r^{n-1} d r\right)^{d / n} \\
& \leq C\left(\int_{0}^{\pi / 2} f(\cos r) \sin ^{n-1} r d r\right)^{d / n} \\
& \leq C\|f\|_{L^{\frac{n}{d}, 1}\left(S^{n}\right)}
\end{aligned}
$$


We will now prove the case $d=1$. From (4.67) we have

$$
\cos s A_{1}^{+} f(s)=C \cos s \int_{0}^{\cos s} f(t)\left(\cos ^{2} s-t^{2}\right)^{-\frac{1}{2}} d t .
$$

Using the change of variable $t=r \cos s$ we have

$$
\begin{aligned}
\cos s A_{1}^{+} f(s) & =C \cos s \int_{0}^{1} f(r \cos s)\left(1-r^{2}\right)^{-\frac{1}{2}} d r \\
& \leq C \cos s \int_{0}^{1} f(r \cos s)(1-r)^{-\frac{1}{2}} d r .
\end{aligned}
$$

For $f(s)=\chi_{E}(s)$ let,

$$
I(s)=\cos s A_{1}^{+}\left(\chi_{E}\right)(s) .
$$

Using (4.73) we get

$$
I(s) \asymp C\left\{\begin{array}{l}
0, \quad \text { if } \cos s<a_{1} . \\
\cos s\left[\sum_{i=1}^{j-1} \int_{a_{i} / \cos s}^{b_{i} / \cos s}(1-r)^{-\frac{1}{2}} d r+\int_{a_{j} / \cos s}^{1}(1-r)^{-\frac{1}{2}} d r\right], \\
\quad \text { ff } a_{j} \leq \cos s<b_{j}, 1 \leq j \leq m . \\
\cos s\left[\sum_{i=1}^{j} \int_{a_{i} / \cos s}^{b_{i} / \cos s}(1-r)^{-\frac{1}{2}} d r\right], \\
\quad \text { if } b_{j} \leq \cos s<a_{j+1}, 1 \leq j \leq m-1 . \\
\cos s\left[\sum_{i=1}^{m} \int_{a_{i} / \cos s}^{b_{i} / \cos s}(1-r)^{-\frac{1}{2}} d r\right], \quad \text { if } b_{m}<\cos s<1 . \\
\left.\quad \begin{array}{c}
\operatorname{if} \cos s<a_{1} \cdot \\
\cos s\left[\sum_{i=1}^{j-1}\left(1-\frac{a_{i}}{\cos s}\right)^{\frac{1}{2}}-\left(1-\frac{b_{i}}{\cos s}\right)^{\frac{1}{2}}+\left(1-\frac{a_{j}}{\cos s}\right)^{\frac{1}{2}}\right] \\
\quad \text { if } a_{j} \leq \cos s<b_{j}, 1 \leq j \leq m . \\
\cos s\left[\sum_{i=1}^{j}\left(1-\frac{a_{i}}{\cos s}\right)^{\frac{1}{2}}-\left(1-\frac{b_{i}}{\cos s}\right)^{\frac{1}{2}}\right], \\
\quad \text { if } b_{j} \leq \cos s<a_{j+1}, 1 \leq j \leq m-1 . \\
\cos s\left[\sum_{i=1}^{m}\left(1-\frac{a_{i}}{\cos s}\right)^{\frac{1}{2}}-\left(1-\frac{b_{i}}{\cos s}\right)^{\frac{1}{2}}\right],
\end{array}\right]
\end{array}\right.
$$

By Lemma 2.1 (with $\gamma=2$ ) we have

$$
I^{2}(s) \leq C\left\{\begin{aligned}
0, \quad & \text { if } \cos s<a_{1} . \\
\cos ^{2} s\left[\sum_{i=1}^{j-1}\left(\frac{b_{i}-a_{i}}{\cos s}\right)+\left(\frac{\cos s-a_{j}}{\cos s}\right)\right], & \quad \text { if } b_{j} \leq \cos s<a_{j+1}, 1 \leq j \leq m-1 . \\
\quad \text { if } a_{j} \leq \cos s<b_{j}, 1 \leq j \leq m . & \text { if } b_{m}<\cos s<1 . \\
\cos ^{2} s\left[\sum_{i=1}^{j}\left(\frac{b_{i}-a_{i}}{\cos s}\right)\right], &
\end{aligned}\right.
$$


Thus we have from above expression that

$$
I^{2}(s) \leq C \sum_{i=1}^{m}\left(b_{i}-a_{i}\right)
$$

We define $A_{i}=1-a_{i}, B_{i}=1-b_{i}, i=1,2, \ldots, m$. Then

$$
A_{1} \geq B_{1} \geq A_{2} \geq B_{2} \cdots \geq A_{m} \geq B_{m} .
$$

Again using the lemma 2.1 (with $\gamma=n / 2$ ) we get

$$
\begin{aligned}
I^{2}(s) & \leq C \sum_{i=1}^{m}\left(b_{i}-a_{i}\right) \\
& =C \sum_{i=1}^{m}\left(A_{i}-B_{i}\right) \\
& \leq C\left[\sum_{i=1}^{m}\left(A_{i}^{\frac{n}{2}}-B_{i}^{\frac{n}{2}}\right)\right]^{2 / n} \\
& =C\left[\sum_{i=1}^{m}\left\{\left(1-a_{i}\right)^{\frac{n}{2}}-\left(1-b_{i}\right)^{\frac{n}{2}}\right\}\right]^{2 / n} \\
& =C\left(\int_{0}^{1} \chi_{E}(s)(1-s)^{\frac{n-2}{2}} d s\right)^{2 / n} \\
& \asymp C\left(\int_{0}^{1} \chi_{E}(s)\left(1-s^{2}\right)^{\frac{n-2}{2}} d s\right)^{2 / n} .
\end{aligned}
$$

Using the change of variable $\cos t=s$, it follows that

$$
I(s) \leq C\left(\int_{0}^{\pi / 2} \chi_{E}(\cos t) \sin ^{n-1} t d t\right)^{1 / n}=C\left\|\chi_{E}\right\|_{L^{n, 1}\left(S^{n}\right)} .
$$

This completes the proof.

The following corollary can be thought of as an analogue of (3.53).

Corollary 4.8. There exists a constant $C>0$ such that for all $f \in L^{\frac{n}{d}, 1}\left(S^{n}\right)$,

$$
\left\|A_{d}^{+} f\right\|_{L^{d+1, \infty}\left(G_{n+1, d+1}\right)} \leq C\|f\|_{L^{\frac{n}{d}, 1}\left(S^{n}\right)} .
$$

Proof. We have from (4.72) that $\left\|A_{d}^{+} f\right\|_{L^{\infty}\left(G_{n+1, d+1}\right)} \leq \frac{C}{\cos \theta}\|f\|_{L^{\frac{n}{d}, 1}\left(S^{n}\right)}$. Since the function $g(\theta)=\frac{1}{\cos \theta}$ belongs to the space $L^{d+1, \infty}\left[0, \frac{\pi}{2}\right]$ with respect to the measure $\cos ^{d} \theta d \theta$ the result follows.

Remark 4.9. i) Interpolating between the estimates (4.72) and (4.69) (for $p=1$ ) we get the following weighted estimate which is somewhat analogous to $\mathbb{R}^{n}$ :

$$
\left\|\cos (\cdot) A_{d}^{+} f\right\|_{L^{q}\left(G_{n+1, d+1}\right)} \leq C\|f\|_{L^{p}\left(S^{n}\right)},
$$

where

$$
1 \leq p<\frac{n}{d}, \quad \frac{n}{p}=\frac{n-d}{q}+d .
$$


ii) Corollary 4.8 has an interesting implication. Suppose that $d+1>\frac{n}{d}$, for instance, we can choose $d=n-1, n>2$. We can now use an interpolation argument involving the estimates $\left\|A_{d}^{+} f\right\|_{L^{1}\left(G_{n+1, d+1}\right)} \leq$ $C\|f\|_{L^{1}\left(S^{n}\right)}$ and (4.79). As a result we can prove that for each $p \in\left(1, \frac{n}{d}\right)$ there exist a $q>p$ such that,

$$
\left\|A_{d}^{+} f\right\|_{L^{q}\left(G_{n+1, d+1}\right)} \leq C\|f\|_{L^{p}\left(S^{n}\right)} .
$$

This implies that for radial functions Theorem 4.5 is not best possible.

Example 4.10. We now construct an example to show that (4.72) is not possible, if $1 \leq p<\frac{n}{d}$. We consider the sequence $\left\{a_{m}\right\}$ where $a_{m}=(m-1) /(m+1)$. Then $\left\{a_{m}\right\}$ is an increasing sequence with $\lim _{m \rightarrow \infty} a_{m}=1$. We define a sequence of functions $\left\{\tilde{f_{m}}\right\}$ by defining $\tilde{f_{m}}(s)=\chi_{\left[a_{m}, 1\right]}(s)$. We now consider the sequence of radial functions on $S^{n}$ defined by $f_{m}\left(\rho \sin \theta+x_{0} \cos \theta\right)=\tilde{f_{m}}(\cos \theta), 0 \leq \theta \leq$ $\pi / 2$. For $\pi / 2<\theta<\pi$, we define $f_{m}$ by the relation $f_{m}(x)=f_{m}(-x)$. We have

$$
\left\|f_{m}\right\|_{L^{p, 1}\left(S^{n}\right)}=C\left(\int_{0}^{\pi / 2} \chi_{\left[a_{m}, 1\right]}(\cos s) \sin ^{n-1} s d s\right)^{1 / p}
$$

By the change of variable $\cos s=t$ in (4.80), we get

$$
\begin{aligned}
\left\|f_{m}\right\|_{L^{p, 1}\left(S^{n}\right)} & =C\left(\int_{0}^{1} \chi_{\left[a_{m}, 1\right]}(t)\left(1-t^{2}\right)^{\frac{n-2}{2}} d t\right)^{1 / p} \\
& \asymp C\left(\int_{0}^{1} \chi_{\left[a_{m}, 1\right](t)}(1-t)^{\frac{n-2}{2}} d t\right)^{1 / p} \\
& =C\left(1-a_{m}\right)^{\frac{n}{2 p}} \\
& =C(m+1)^{-\frac{n}{2 p}} .
\end{aligned}
$$

If $\frac{(m+1) a_{m}}{m}<\cos s<\frac{m a_{m}}{m-1}$, then it follows from the explicit expression of the Abel transform (see (4.67)) that

$$
\begin{aligned}
\cos s A_{d}^{+} f_{m}(s) & =\frac{C}{\cos ^{d-1} s} \int_{0}^{\cos s} f_{m}(t)\left(\cos ^{2} s-t^{2}\right)^{\frac{d-2}{2}} d t \\
& =\frac{C}{\cos ^{d-1} s} \int_{a_{m}}^{\cos s}\left(\cos ^{2} s-t^{2}\right)^{\frac{d-2}{2}} d t .
\end{aligned}
$$

By substituting $t=r \cos s$ in (4.82) we get

$$
\begin{aligned}
\cos s A_{d}^{+} f_{m}(s) & =C \int_{\frac{a_{m}}{\cos s}}^{1}\left(1-r^{2}\right)^{\frac{d-2}{2}} d r \\
& \geq C\left(1-\frac{a_{m}}{\cos s}\right)^{\frac{d}{2}} \\
& \geq C\left(1-\frac{m}{m+1}\right)^{\frac{d}{2}} \\
& =C(m+1)^{-\frac{d}{2}} .
\end{aligned}
$$

It follows from (4.81) and (4.83) that an estimate of the form

$$
\left\|\cos (\cdot) A_{d}^{+} f(\cdot)\right\|_{L^{\infty}\left(G_{n+1, d+1}\right)} \leq C\|f\|_{L^{p, 1}\left(S^{n}\right)}
$$

is possible only if $\frac{n}{2 p} \leq \frac{d}{2}$, that is, $p \geq \frac{n}{d}$. 


\section{REFERENCES}

[1] Carlos A. Berenstein and Boris Rubin, Radon transform of $L^{p}$-functions on the Lobachevsky space and hyperbolic wavelet transforms. Forum Math. no. 5, 11 (1999), 567-590.

[2] Carlos A. Berenstein and Boris Rubin, Totally geodesic Radon transform of $L^{p}$-functions on real hyperbolic space. Fourier analysis and convexity, 37-58, Appl. Numer. Harmon. Anal., Birkhuser Boston, Boston, MA, (2004).

[3] William O. Bray, Aspects of harmonic analysis on real hyperbolic space. Fourier analysis (Orono, ME, 1992), 77-102.

[4] Michael Cowling, Stefano Meda and Alberto G. Setti, An overview of harmonic analysis on the group of isometries of a homogeneous tree. Exposition. Math. 16 (1998), no. 5, 385-423.

[5] Javier Duoandikoetxea, Virginia Naibo and Osane Oruetxebarria, $k$-plane transforms and related operators on radial functions. Michigan Math. J. 49 (2001), 265-276.

[6] L. Grafakos, Classical and modern Fourier analysis, (2004), Pearson Education, Inc. NJ.

[7] Fulton B. Gonzalez, Radon transform on Grassmann manifolds. Journal of Func. Anal., bf 71 (1987), 339-362.

[8] Fulton B. Gonzalez and Tomoyuki Kakehi, Pfaffian systems and Radon transforms on affine Grassmann manifolds. Math. Ann., 326 (2003), no. 2, 237-273.

[9] Fulton B. Gonzalez and Tomoyuki Kakehi, Dual Radon transforms on affine Grassmann manifolds. Trans. Amer. Math. Soc. 356 (2004), no. 10, 4161-4180

[10] Sigurdur Helgason, The Radon transform on Euclidean spaces, compact two-point homogeneous spaces and Grassmann manifolds. Acta Math., 113 (1965), 153-180.

[11] Sigurdur Helgason, The Radon transform. Second edition. Progress in Mathematics, 5. Birkhuser Boston, Inc., Boston, MA, (1999).

[12] Sigurdur Helgason, Groups and geometric analysis. Integral geometry, invariant differential operators, and spherical functions. Mathematical Surveys and Monographs, 83. American Mathematical Society, Providence, RI, (2000).

[13] Alexandru D. Ionescu, An endpoint estimate for the Kunze-Stein phenomenon and related maximal operators. Ann. of Math. (2) 152 (2000), no. 1, 259-275.

[14] Satoshi Ishikawa, The range characterizations of the totally geodesic Radon transform on the real hyperbolic space. Duke Math. J. 90 (1997), no. 1, 149-203.

[15] Ashisha Kumar and Swagato K. Ray, Weighted estimates of d-plane transform for radial functions on Euclidean spaces. Israel J. Math. 188 (2012), no. 1, 25-56.

[16] Andrew Markoe, Analytic tomography. Encyclopedia of Mathematics and its Applications, 106. Cambridge University Press, Cambridge, (2006).

[17] D. M. Oberlin and E. M. Stein, Mapping properties of the Radon transform. Indiana Univ. Math. J. 31 (1982), 641-650.

[18] John G. Ratcliffe, Foundations of hyperbolic manifolds. Second edition. Graduate Texts in Mathematics, 149. Springer, New York, (2006).

[19] Swagato K. Ray and Rudra P. Sarkar, Fourier and Radon transform on harmonic NA groups. Trans. Amer. Math. Soc. 361 (2009), no. 8, 4269-4297.

[20] Boris Rubin, Inversion formulas for the spherical Radon transform and the generalized cosine transform. Adv. in Appl. Math. 29 (2002), no. 3, 471-497.

[21] Boris Rubin, Radon, cosine and sine transforms on real hyperbolic space. Adv. Math. 170 (2002), no. 2, $206-223$.

[22] Boris Rubin, Radon transforms on affine Grassmannians. Trans. Amer. Math. Soc. 356 (2004), no. 12, 5045-5070.

[23] Donald C. Solmon, A note on k-plane integral transforms. J. Math. Anal. Appl. 71 (1979), 351-358.

[24] Robert S. Strichartz, Harmonic analysis on Grassmannian bundles. Trans. of the Amer. Math. Soc., 296 (1986), 387-409.

[25] Robert S. Strichartz, $L^{p}$ estimates for Radon transforms in Euclidean and non-Euclidean spaces. Duke Math. J. 48 (1981), no. 4, 699-727.

[26] E. M. Stein and G. Weiss, Introduction to Fourier analysis on Euclidean spaces. Princeton Mathematical Series, No. 32. Princeton University Press, Princeton, N.J., (1971).

[27] N. Ja. Vilenkin and A. U. Klimyk, Representation of Lie groups and special functions. Vol. 2. Class I representations, special functions, and integral transforms. Translated from the Russian by V. A. Groza and A. A. Groza. Mathematics and its Applications (Soviet Series), 74. Kluwer Academic Publishers Group, Dordrecht, (1993).

(Ashisha Kumar) Department of mathematics, Indian Institute of Science, Bangalore 560012, Karnatka, INDIA, E-MAIL: ASHISHA10@MATH.IISC.ERNET.IN

(Swagato K. Ray) Stat-math Unit, Indian Statistical Institute, 203 B. T. Road, Kolkata 700108, India, E-MAIL:SKRAY@IITK.AC.IN 